\title{
Management of Sucking Pest of Groundnut with Newer Molecules of Insecticides (Arachis hypogaea L.)
}

\author{
V.K. Nigude, S.P. Patil, S.A. Patil and A.S. Bagade* \\ Department of Agril. Entomology, College of Agriculture Kolhapur - 416004 , \\ Maharashtra, India \\ *Corresponding author
}

\section{A B S T R A C T}

\begin{tabular}{|l|}
\hline K e y w o r d s \\
Groundnut, Jassids, \\
Thrips, Bio-efficacy \\
\hline Article Info \\
\hline $\begin{array}{l}\text { Accepted: } \\
\text { 06 December } 2017 \\
\text { Available Online: } \\
\text { 10 January 2018 }\end{array}$ \\
\hline
\end{tabular}

\section{Keywords}

Groundnut, Jassids, Thrips, Bio-efficacy

\section{Article Info}

06 December 2017

10 January 2018

\begin{abstract}
Bio-efficacy of newer molecules of insecticides against sucking pests of groundnut (Arachis hypogaea L.) was studied during July to October 2016, at Reasearch farm, College of agriculture, Kolhapur. Groundnut crop was infested by Jassid (Empoasca kerri Pruthi), Thrips (Scirtotharips dorsalis Hood). The experiment was laid out in randomized block design with eight treatment and three replications with view to find out the effective insecticides against sucking pest of groundnut at Agronomy field, college of agriculture Kolhapur during Rabi 2016-17. Variety TAG-24 was sown on $1^{\text {st }}$ July. The insecticide used for the treatment were quinalphos $25 \mathrm{EC}$, acephate $75 \mathrm{SP}$, imidacloprid 17.8 SL, lambda-cyhalothrin $5 \mathrm{EC}$, methomyl $40 \mathrm{SP}$, buprofenzin $25 \mathrm{SC}$, chlorpyriphos $20 \mathrm{EC}$. The results of present investigation revealed that the treatment with imidacloprid 17.8 SL was significantly most effective treatment for the control of sucking pest.
\end{abstract}

\section{Introduction}

Groundnut (Arachis hypogaea L.) is an annual legume crop, also known as peanut, earthnut, monkey- nut and goobers. It forms the world's largest source of edible oil and ranks $13^{\text {th }}$ among the food crops and is also $4^{\text {th }}$ most important oilseed crop of the world. It is grown in tropical and subtropical countries. Cultivated groundnut has been reported from South America (Weiss, 2000). It is grown in 26.4 million hectares with a production of 38.20 million tones and productivity of $691 \mathrm{~kg}$ $\mathrm{ha}^{-1}(\mathrm{FAO}, 2010)$. Asia posses $1^{\text {st }}$ rank in area $(63.4 \%)$ and production (71.1\%). Major groundnut countries are India (26\%), China
$(19 \%)$ and Nigeria (11\%). In India is mainly grown in the southern and north-western states; Gujrat, Andhra Pradesh, Tamil nadu, Karnataka, Maharastra, and Madhya Pradesh, together occupying about 90 percent of the groundnut area in the country. The major insect pest of groundnut are groundnut aphid (Aphis craccivora Koch), leaf miner (Stomopteryx nertara meyrick), stem borer (Sphenoptera perotett camron), white grub (Holotrichia consainguinia Blanchard), Bihar hairy caterpillar (Spilosoma oblique walker), Tobbaco caterpillar (Spodoptera litura Fab.), Red hairy caterpillar (Amsacta albistriga Butler), Jassid (Empoasca kerri Pruthi), Thrips (Scritothrips dorsalis), Termite 
(Odontotermes obesus Rambur) as reported Atwal and Dhaliwal (2008). However, aphid was not considerd to be a serious pest of groundnut until late 1980 (Nandgopal, 1992). Mostly, groundnut insect pests are sporadic in occurrence and distribution. However, there are instances of total crop loss caused by a single pest species. Although many insect species live and feed on the groundnut crop, only a few causes significant damage that result in large reductions in pod and haulm yields.

\section{Materials and Methods}

The experiment was laid in simple randomized block design with eight treatments and three replications. Net plot size was $3.00 \times 1.80 \mathrm{~m}^{2}$. Row to row and plant to plant spacing was 30 $\mathrm{cm}$ and $10 \mathrm{~cm}$, respectively. Observations were recorded after 3,7 and 10 days of spraying on sucking pest infesting groundnut.

\section{Jassids, Empoasca kerri (Pruthi)}

Numbers of jassids on three compound leaves (upper, middle and lower) per plant were recorded from selected plants from each of the five plants as per the method suggested by Yeotiker et al., (2015).

\section{Thrips, [Scirtothrips dorsalis (Hood)]}

Numbers of jassids on three compound leaves (upper, middle and lower) per plant were recorded from selected plants from each of the five plants as per the method suggested by Yeotiker et al., (2015)

Table.1 Bio-efficacy of newer molecules of insecticides against jassids on groundnut

\begin{tabular}{|c|c|c|c|c|c|c|c|c|c|}
\hline \multirow{3}{*}{ Tr.No } & \multirow{3}{*}{ Treatments } & \multicolumn{8}{|c|}{ Mean survival population of Jassids/ 3leaves } \\
\hline & & \multicolumn{4}{|c|}{ First Spraying } & \multicolumn{4}{|c|}{ Second Spraying } \\
\hline & & $\begin{array}{c}3 \\
\text { DAS }\end{array}$ & $\begin{array}{c}7 \\
\text { DAS }\end{array}$ & 10 DAS & Mean & $\begin{array}{c}3 \\
\text { DAS }\end{array}$ & $\begin{array}{c}7 \\
\text { DAS }\end{array}$ & 10 DAS & Mean \\
\hline T1 & Quinalphos $25 \mathrm{EC}$ & $\begin{array}{c}2.14 \\
(1.63)\end{array}$ & $\begin{array}{c}2.94 \\
(1.86)\end{array}$ & $\begin{array}{l}\mathbf{3 . 1 9} \\
(1.92)\end{array}$ & $\begin{array}{c}2.75 \\
(1.80)\end{array}$ & $\begin{array}{c}2.28 \\
(1.67)\end{array}$ & $\begin{array}{c}\mathbf{2 . 8 0} \\
(1.82)\end{array}$ & $\begin{array}{c}2.97 \\
(1.86)\end{array}$ & $\begin{array}{c}\mathbf{2 . 6 8} \\
(1.78)\end{array}$ \\
\hline $\mathbf{T 2}$ & Acephate 75 SP & $\begin{array}{c}1.20 \\
(1.30)\end{array}$ & $\begin{array}{c}1.63 \\
(1.46)\end{array}$ & $\begin{array}{c}2.49 \\
(1.73)\end{array}$ & $\begin{array}{c}1.77 \\
(1.49)\end{array}$ & $\begin{array}{c}\mathbf{1 . 2 6} \\
(1.32)\end{array}$ & $\begin{array}{c}\mathbf{1 . 7 9} \\
(1.51)\end{array}$ & $\begin{array}{c}2.37 \\
(1.69)\end{array}$ & $\begin{array}{c}1.80 \\
(1.50)\end{array}$ \\
\hline T3 & Imidacloprid 17.8 SL & $\begin{array}{c}\mathbf{0 . 5 6} \\
(1.03)\end{array}$ & $\begin{array}{c}1.08 \\
(1.26)\end{array}$ & $\begin{array}{c}\mathbf{1 . 8 6} \\
(1.54)\end{array}$ & $\begin{array}{c}\mathbf{1 . 1 6} \\
(1.27)\end{array}$ & $\begin{array}{c}\mathbf{0 . 7 3} \\
(1.11)\end{array}$ & $\begin{array}{c}\mathbf{1 . 2 1} \\
(1.31)\end{array}$ & $\begin{array}{c}1.93 \\
(1.56)\end{array}$ & $\begin{array}{c}1.29 \\
(1.32)\end{array}$ \\
\hline T4 & $\begin{array}{l}\text { Lambda-cyhalothrin } \\
5 \mathrm{EC}\end{array}$ & $\begin{array}{c}\mathbf{0 . 8 9} \\
(1.18)\end{array}$ & $\begin{array}{c}1.47 \\
(1.40)\end{array}$ & $\begin{array}{c}\mathbf{2 . 3 8} \\
(1.70)\end{array}$ & $\begin{array}{c}\mathbf{1 . 5 8} \\
(1.42)\end{array}$ & $\begin{array}{c}\mathbf{1 . 0 3} \\
(1.23)\end{array}$ & $\begin{array}{c}1.63 \\
(1.46)\end{array}$ & $\begin{array}{c}2.34 \\
(1.68)\end{array}$ & $\begin{array}{c}1.66 \\
(1.45)\end{array}$ \\
\hline T5 & Methomyl 40 SP & $\begin{array}{c}\mathbf{1 . 2 8} \\
(1.33)\end{array}$ & $\begin{array}{c}\mathbf{1 . 7 8} \\
(1.51)\end{array}$ & $\begin{array}{c}\mathbf{2 . 6 4} \\
(1.77)\end{array}$ & $\begin{array}{c}\mathbf{1 . 9 0} \\
(1.53)\end{array}$ & $\begin{array}{c}\mathbf{1 . 3 9} \\
(1.37)\end{array}$ & $\begin{array}{c}\mathbf{1 . 8 4} \\
(1.53)\end{array}$ & $\begin{array}{c}\mathbf{2 . 4 2} \\
(1.71)\end{array}$ & $\begin{array}{c}\mathbf{1 . 8 8} \\
(1.53)\end{array}$ \\
\hline T6 & Buprofenzin $25 \mathrm{SC}$ & $\begin{array}{c}\mathbf{2 . 1 2} \\
(1.62)\end{array}$ & $\begin{array}{c}\mathbf{2 . 8 8} \\
(1.84)\end{array}$ & $\begin{array}{c}\mathbf{3 . 1 1} \\
(1.90)\end{array}$ & $\begin{array}{c}\mathbf{2 . 7 0} \\
(1.78)\end{array}$ & $\begin{array}{c}\mathbf{2 . 2 3} \\
(1.65)\end{array}$ & $\begin{array}{c}\mathbf{2 . 7 3} \\
(1.80)\end{array}$ & $\begin{array}{c}\mathbf{2 . 8 9} \\
(1.84)\end{array}$ & $\begin{array}{c}\mathbf{2 . 6 1} \\
(1.76)\end{array}$ \\
\hline T7 & Chlorpyriphos $20 \mathrm{EC}$ & $\begin{array}{c}\mathbf{2 . 8 9} \\
(1.84)\end{array}$ & $\begin{array}{c}\mathbf{3 . 0 0} \\
(1.87)\end{array}$ & $\begin{array}{c}3.27 \\
(1.94)\end{array}$ & $\begin{array}{c}\mathbf{3 . 0 5} \\
(1.88)\end{array}$ & $\begin{array}{c}\mathbf{2 . 9 8} \\
(1.87)\end{array}$ & $\begin{array}{c}\mathbf{3 . 0 6} \\
(1.89)\end{array}$ & $\begin{array}{c}\mathbf{3 . 2 2} \\
(1.93)\end{array}$ & $\begin{array}{c}\mathbf{3 . 0 0} \\
(1.89)\end{array}$ \\
\hline T8 & Untreated control & $\begin{array}{c}\mathbf{8 . 8 8} \\
(3.06)\end{array}$ & $\begin{array}{c}9.08 \\
(3.10)\end{array}$ & $\begin{array}{c}\mathbf{8 . 9 2} \\
(3.07)\end{array}$ & $\begin{array}{c}8.96 \\
(3.07)\end{array}$ & $\begin{array}{c}\mathbf{8 . 9 7} \\
(3.08)\end{array}$ & $\begin{array}{c}9.16 \\
(3.11)\end{array}$ & $\begin{array}{c}\mathbf{8 . 9 9} \\
(3.08)\end{array}$ & $\begin{array}{c}9.04 \\
(3.09)\end{array}$ \\
\hline & $\mathrm{SE} \pm$ & 0.06 & 0.02 & 0.03 & & 0.04 & 0.05 & 0.03 & \\
\hline & CD at $\overline{5} \%$ & 0.13 & 0.07 & 0.11 & & 0.11 & 0.13 & 0.09 & \\
\hline
\end{tabular}

DAS $=$ Days after spray

*Figures in parentheses are transformed values $\sqrt{\boldsymbol{X}+\mathbf{0 . 5}}$ 
Table.2 Bio-efficacy of newer molecules of insecticides against thrips on groundnut

\begin{tabular}{|c|c|c|c|c|c|c|c|c|c|}
\hline \multirow{3}{*}{ Tr.No } & \multirow{3}{*}{ Treatments } & \multicolumn{8}{|c|}{ Mean survival population of thrips/3leaves } \\
\hline & & \multicolumn{4}{|c|}{ First Spraying } & \multicolumn{4}{|c|}{ Second Spraying } \\
\hline & & $\begin{array}{c}3 \\
\text { DAS }\end{array}$ & $\begin{array}{c}7 \\
\text { DAS }\end{array}$ & 10 DAS & Mean & $\begin{array}{c}3 \\
\text { DASS }\end{array}$ & $\begin{array}{c}7 \\
\text { DAS }\end{array}$ & 10 DAS & Mean \\
\hline T1 & Quinalphos $25 \mathrm{EC}$ & $\begin{array}{c}\mathbf{1 . 4 9} \\
(1.71)\end{array}$ & $\begin{array}{c}\mathbf{1 . 8 5} \\
(1.54)\end{array}$ & $\begin{array}{c}\mathbf{2 . 0 3} \\
(1.59)\end{array}$ & $\begin{array}{c}\mathbf{1 . 7 9} \\
(1.61)\end{array}$ & $\begin{array}{c}\mathbf{1 . 5 9} \\
(1.45)\end{array}$ & $\begin{array}{c}\mathbf{1 . 9 6} \\
(1.57)\end{array}$ & $\begin{array}{c}\mathbf{2 . 1 4} \\
(1.63)\end{array}$ & $\begin{array}{c}\mathbf{1 . 8 9} \\
(1.55)\end{array}$ \\
\hline T2 & Acephate 75 SP & $\begin{array}{c}\mathbf{1 . 1 5} \\
(1.28)\end{array}$ & $\begin{array}{c}\mathbf{1 . 2 9} \\
(1.34)\end{array}$ & $\begin{array}{c}\mathbf{1 . 5 3} \\
(1.43)\end{array}$ & $\begin{array}{c}\mathbf{1 . 3 2} \\
(1.35)\end{array}$ & $\begin{array}{c}\mathbf{1 . 2 2} \\
(1.31)\end{array}$ & $\begin{array}{c}\mathbf{1 . 4 9} \\
(1.41)\end{array}$ & $\begin{array}{c}\mathbf{1 . 6 8} \\
(1.47)\end{array}$ & $\begin{array}{c}\mathbf{1 . 4 6} \\
(1.39)\end{array}$ \\
\hline T3 & Imidacloprid 17.8 SL & $\begin{array}{c}\mathbf{0 . 7 9} \\
(1.14)\end{array}$ & $\begin{array}{c}\mathbf{0 . 9 5} \\
(1.20)\end{array}$ & $\begin{array}{c}\mathbf{1 . 1 7} \\
(1.29)\end{array}$ & $\begin{array}{c}\mathbf{0 . 9 7} \\
(1.21)\end{array}$ & $\begin{array}{c}\mathbf{0 . 9 0} \\
(1.18)\end{array}$ & $\begin{array}{c}\mathbf{1 . 2 0} \\
(1.30)\end{array}$ & $\begin{array}{l}\mathbf{1 . 3 0} \\
(1.34)\end{array}$ & $\begin{array}{c}\mathbf{1 . 1 3} \\
(1.27)\end{array}$ \\
\hline T4 & $\begin{array}{l}\text { Lambda-cyhalothrin } \\
5 \text { EC }\end{array}$ & $\begin{array}{l}\mathbf{1 . 0 6} \\
(1.24)\end{array}$ & $\begin{array}{c}\mathbf{1 . 2 3} \\
(1.32)\end{array}$ & $\begin{array}{c}\mathbf{1 . 4 4} \\
(1.39)\end{array}$ & $\begin{array}{c}\mathbf{1 . 2 4} \\
(1.31)\end{array}$ & $\begin{array}{c}\mathbf{1 . 1 6} \\
(1.29)\end{array}$ & $\begin{array}{c}\mathbf{1 . 4 5} \\
(1.40)\end{array}$ & $\begin{array}{c}\mathbf{1 . 6 2} \\
(1.46)\end{array}$ & $\begin{array}{c}\mathbf{1 . 4 1} \\
(1.38)\end{array}$ \\
\hline T5 & Methomyl 40 SP & $\begin{array}{c}\mathbf{1 . 1 8} \\
(1.30)\end{array}$ & $\begin{array}{c}\mathbf{1 . 3 6} \\
(1.36)\end{array}$ & $\begin{array}{c}\mathbf{1 . 5 8} \\
(1.44)\end{array}$ & $\begin{array}{c}\mathbf{1 . 3 7} \\
(1.36)\end{array}$ & $\begin{array}{c}\mathbf{1 . 2 7} \\
(1.33)\end{array}$ & $\begin{array}{c}\mathbf{1 . 5 6} \\
(1.44)\end{array}$ & $\begin{array}{c}\mathbf{1 . 7 3} \\
(1.49)\end{array}$ & $\begin{array}{c}\mathbf{1 . 5 2} \\
(1.42)\end{array}$ \\
\hline T6 & Buprofenzin $25 \mathrm{SC}$ & $\begin{array}{c}\mathbf{1 . 4 6} \\
(1.62)\end{array}$ & $\begin{array}{c}\mathbf{1 . 7 9} \\
(1.51)\end{array}$ & $\begin{array}{c}\mathbf{1 . 9 8} \\
(1.57)\end{array}$ & $\begin{array}{c}\mathbf{1 . 7 4} \\
(1.56)\end{array}$ & $\begin{array}{c}\mathbf{1 . 5 2} \\
(1.42)\end{array}$ & $\begin{array}{c}\mathbf{1 . 8 8} \\
(1.54)\end{array}$ & $\begin{array}{c}\mathbf{2 . 0 8} \\
(1.61)\end{array}$ & $\begin{array}{c}\mathbf{1 . 8 2} \\
(1.52)\end{array}$ \\
\hline T7 & Chlorpyriphos $20 \mathrm{EC}$ & $\begin{array}{c}\mathbf{1 . 5 5} \\
(1.90)\end{array}$ & $\begin{array}{c}\mathbf{2 . 0 2} \\
(1.59)\end{array}$ & $\begin{array}{c}\mathbf{2 . 1 6} \\
(1.63)\end{array}$ & $\begin{array}{c}\mathbf{1 . 9 1} \\
(1.71)\end{array}$ & $\begin{array}{c}\mathbf{1 . 6 4} \\
(1.46)\end{array}$ & $\begin{array}{c}\mathbf{2 . 0 4} \\
(1.60)\end{array}$ & $\begin{array}{l}\mathbf{2 . 2 0} \\
(1.64)\end{array}$ & $\begin{array}{c}\mathbf{1 . 9 6} \\
(1.56)\end{array}$ \\
\hline T8 & Untreated control & $\begin{array}{c}\mathbf{8 . 6 0} \\
(3.02)\end{array}$ & $\begin{array}{c}9.08 \\
(3.10)\end{array}$ & $\begin{array}{c}\mathbf{8 . 9 8} \\
(3.08)\end{array}$ & $\begin{array}{c}\mathbf{8 . 8 8} \\
(3.06)\end{array}$ & $\begin{array}{c}\mathbf{7 . 1 5} \\
(2.77)\end{array}$ & $\begin{array}{c}\mathbf{6 . 3 8} \\
(2.62)\end{array}$ & $\begin{array}{l}7.05 \\
(2.75)\end{array}$ & $\begin{array}{c}\mathbf{6 . 8 6} \\
(2.71)\end{array}$ \\
\hline & $\mathrm{SE} \pm$ & 0.02 & 0.03 & 0.02 & & 0.03 & 0.02 & 0.04 & \\
\hline & CD at $5 \%$ & 0.07 & 0.09 & 0.08 & & 0.10 & 0.08 & 0.11 & \\
\hline
\end{tabular}

DAS $=$ Days after spray

*Figures in parentheses are transformed values $\sqrt{\boldsymbol{X}+\mathbf{0 . 5}}$

\section{Results and Discussion}

\section{First spray for jassids}

Overall results on efficacy of treatments indicated that imidacloprid 17.8 SL@0.75 $\mathrm{ml} /$ lit. (1.16 jassids/3 leaves) was consistently most effective as compared to other treatments in reducing the survival population of jassids.

The treatment with lambda-cyhalothrin $5 \mathrm{EC}$ @ $0.50 \mathrm{ml} /$ lit (1.58 jassids/3 leaves) was the next best effective treatment, followed by acephate 75 SP @ 1.0 gm/lit (1.77 jassids/3 leaves).

\section{Second spray for jassids}

Overall results on efficacy of treatments indicated that imidacloprid 17.8 SL@0.75 $\mathrm{ml} /$ lit (1.29 jassids/3 leaves) was found to be the most effective treatment compared to other insecticidal treatments in reducing the survival population of jassids. The treatment with lambda-cyhalothrin 5 EC @ $0.50 \mathrm{ml} / \mathrm{lit}$. (1.66 jassids/3 leaves) was the next best effective treatment, followed by acephate 75 SP @ 1.0 gm/lit (1.80 jassids/3 leaves) (Table $1)$.

\section{First spray for thrips}

Overall results on efficacy indicated that imidacloprid 17.8 SL @ $0.75 \mathrm{ml} / \mathrm{lit}(0.97$ thrips/3 leaves) was the consistently most effective compared to other treatments in reducing the survival population of thrips. The treatment with lambda-cyhalothrin 5 EC @ $0.50 \mathrm{ml} / \mathrm{lit}$ (1.24 thrips/3 leaves) was the next best effective treatment, closely followed by acephate $75 \mathrm{SP} @ 1.0 \mathrm{gm} / \mathrm{lit}$ (1.32 thrips/3 leaves) (Table 2). 


\section{Second spray for thrips}

Overall results on efficacy indicated that imidacloprid 17.8 SL @0.75 ml/lit (1.13 thrips/3 leaves) was the most effective as compared to other treatments in reducing the survival population of thrips. The treatment with lambda-cyhalothrin 5 EC @ $0.50 \mathrm{ml} / \mathrm{lit}$ (1.41 thrips/3 leaves) was the next best effective treatment, closely followed by acephate 75 SP @ 1.0 gm/lit (1.46 thrips/3 leaves).

\section{References}

Atwal, A.S. and Dhaliwal, G. S. 2008. Agricultural pests of south Asia and their management. Publ. Rajender nagar, Ludhiana, pp. 274-277

FAO 2010. FAO Production Year book, Vol. 60.

Nandgopal, V. 1992. Studied on integrated pest management in groundnut in Saurastra Ph.D. thesis submitted to Saurasta University, Rajkot, Pesticides, 8:246.

Weiss, E. A. 2000. Oilseed Crops. Publ. by Blackwell Science, London. p. 13.

Yeotikar, S. G., More, D. G. and Gaikwad, B. B. 2015. Seasonal incidence of major insect pests of soybean. Indian J. agric. Sci., 39(4): 341-346.

\section{How to cite this article:}

Nigude, V.K., S.P. Patil, S.A. Patil and Bagade, A.S. 2018. Management of Sucking Pest of Groundnut with Newer Molecules of Insecticides (Arachis hypogaea L.). Int.J.Curr.Microbiol.App.Sci. 7(01): 566-569. doi: https://doi.org/10.20546/ijcmas.2018.701.068 